\title{
Universal Behavior of the Initial Stage of Drop Impact
}

\author{
Evert Klaseboer, ${ }^{1, *}$ Rogerio Manica, ${ }^{1, \dagger}$ and Derek Y. C. Chan ${ }^{1,2,3, \dagger}$ \\ ${ }^{1}$ Institute of High Performance Computing, 1 Fusionopolis Way, 138632 Singapore, Singapore \\ ${ }^{2}$ Department of Mathematics and Statistics, University of Melbourne, Parkville 3010, Australia \\ ${ }^{3}$ Department of Chemistry and Biotechnology, Swinburne University of Technology, Hawthorn 3122, Australia
}

(Received 14 May 2014; published 4 November 2014)

During the early stages of the impact of a drop on a solid surface, pressure builds up in the intervening thin lubricating air layer and deforms the drop. The extent of the characteristic deformation is determined by the competition between capillary, gravitational, and inertial forces that has been encapsulated in a simple analytic scaling law. For millimetric drops, variations of the observed deformation with impact velocity $V$ exhibit a maximum defined by the Weber and Eötvös numbers: We $=1+$ Eo. The deformation scales as $V^{1 / 2}$ at the low-velocity capillary regime and as $V^{-1 / 2}$ at the high-velocity inertia regime, in excellent agreement with a variety of experimental systems.

DOI: 10.1103/PhysRevLett.113.194501

PACS numbers: 47.55.D-, 47.15.gm

The systematic study of drop impact phenomena has a long history [1], with recent experimental [2] and theoretical [3-5] effort focused on the spreading dynamics of the drop. For instance, understanding drop impact phenomena is vital in the application of insecticides and fertilizers in agriculture [6,7]. The improving capabilities of high-speed video recording provided renewed impetus in the subject, allowing the role of the thin air layer trapped between the drop and the target solid to be elucidated under well controlled conditions [8-13]. A recent study of direct observations of the initial drop deformation at impact using high-speed interferometry [14] reported that at a fixed drop size, the extent of dimple deformation upon impact exhibits a maximum as a function of impact velocity. Such a dimple is the precursor to the entrapment of a small air bubble under the center of the impacting drop that can be undesirable in some applications [2]. With a ratio of $\sim 1000$ or more between the drop and bubble size, the challenges in detailed modeling are formidable [3,15].

Here, we show that this dimple formation is a part of a unified phenomenon that results from the competition between capillary, inertial, and to a lesser extent, gravitational forces. Indeed, results from currently available experimental range of impact velocities from around $20 \mu \mathrm{m} / \mathrm{s}$ to $3 \mathrm{~m} / \mathrm{s}$ can be captured accurately by a simple and physically perspicuous analytic formula. Furthermore, dimple formation in bubble-surface collisions as well as drop-drop and drop-surface collision dynamics can also be described quantitatively by the same model under the same theoretical framework. But when inertial effects are dominant, the scaling behavior with impact velocity of $V^{-1 / 2}$ is different from that of the $V^{-2 / 3}$ velocity proposed earlier $[14,16]$. The difference lies in the estimates of the pressure at the bottom of the drop at impact.

Model.-Our analysis uses a model in which a spherical drop of radius $R$, falling under constant gravity $g$ onto a horizontal solid surface is treated as an inviscid fluid of density $\rho_{\text {in }}$, whose dynamics are described by the Bernoulli equation. The deformation of the drop against a constant surface tension $\sigma$ is governed by the normal stress balance, whereas the dynamics of the thin viscous air film of viscosity $\mu_{\text {out }}$ trapped between the bottom of the drop and the target solid is described by incompressible StokesReynolds lubrication theory because the characteristic film Reynolds number is small. Otherwise, outside this film, the air is assumed to have constant pressure $p_{o}$; that is, we omit flow and hydrostatic effects in the air because of its small density and viscosity. Detailed derivations of these governing equations can be found in the Supplemental Material [17]. At impact, we approximate the pressure $p_{B}$ along the bottom of the drop including the stagnation pressure, estimated using the Bernoulli equation (see Fig. 1) [17,18]

$$
p_{B} \simeq p_{o}+\frac{2 \sigma}{R_{T}}+\frac{1}{2} \rho_{\text {in }} V^{2}+\rho_{\text {in }} g z_{B}
$$

where $R_{T}$ is the mean radius of curvature at the top apex of the drop at the initial stage of impact and can be assumed to be equal to the drop radius $R$.

The spatial variation of the thickness $h$ of the air film between the bottom of the drop and the solid surface is given by the normal stress balance. For an axisymmetric drop and film, $h(r, t)$ is given by (see Fig. 1)

$$
\frac{2 \sigma}{R_{B}}=\frac{\sigma}{r} \frac{\partial}{\partial r}\left(r \frac{\partial h}{\partial r}\right)=p_{B}-p_{f}
$$

From Eqs. (1) and (2), the excess pressure, $p_{f}-p_{o}$ that drives the lubricating flow is

$$
p_{f}-p_{o}=\frac{2 \sigma}{R_{T}}-\frac{2 \sigma}{R_{B}}+\frac{1}{2} \rho_{\text {in }} V^{2}+\rho_{\text {in }} g z_{B}
$$




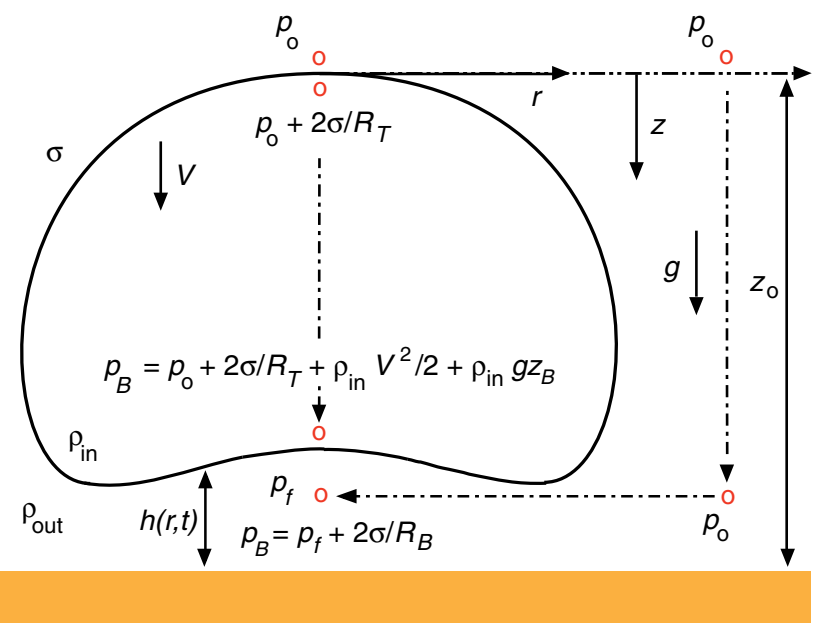

FIG. 1 (color online). Schematic of the drop or bubble impacting on a horizontal solid surface. Assuming the pressure above the drop is $p_{o}$, a pressure jump of $2 \sigma / R_{T}$ will occur when we cross the boundary on top. Since the bottom of the drop hardly moves, but the top still approaches with velocity $V$, a stagnation pressure $\rho_{\text {in }} V^{2} / 2$ will build up in the bottom part of the drop. Alternatively, going around the drop then inside the film will lead to a film pressure $p_{f}$. Finally, $p_{f}$ and $p_{B}$ are related through the normal stress balance (the pressure difference between two sides of the interface is the product of the surface tension and the total curvature).

with $p_{f} \rightarrow p_{o}$ outside the film. As the typical film thickness is on the order of $\mu \mathrm{m}$, we may omit intermolecular forces (e.g., van der Waals attraction) between the drop and the solid surface during the initial stage of drop impact.

Because of viscous drainage of the air layer of viscosity $\mu_{\text {out }}$ between the bottom of the drop and the surface, the time evolution of the thickness of this axisymmetric film is governed by the Stokes-Reynolds lubrication equation [19]

$$
\frac{\partial h}{\partial t}=\frac{1}{12 \mu_{\text {out }}} \frac{1}{r} \frac{\partial}{\partial r}\left(r h^{3} \frac{\partial p_{f}}{\partial r}\right)
$$

where we have assumed that the immobile boundary condition holds at the drop surface because the drop viscosity is much higher than the air viscosity [20]. However, surface active agents can also give rise to an immobile boundary condition [21]. This equation is valid if the film Reynolds number: $\operatorname{Re}_{f}=\rho_{\text {out }} V(H R)^{1 / 2} / \mu_{\text {out }} \ll 1$, based on the radial dimension $r \sim(H R)^{1 / 2}$, with $H$ the characteristic film thickness and $V$ the characteristic impact velocity.

Equations (1), (2), and (4) constitute the model that governs drop dynamics at impact.

Scaling analysis.-From Eq. (3) we see that the characteristic film pressure $P$ has combined contributions from surface tension, gravity, and fluid inertia

$$
P \simeq \frac{\sigma}{R}+\rho_{\text {in }} V^{2}+\rho_{\text {in }} g R .
$$

From Eq. (4), with $r \sim \sqrt{H R}$, we obtain

$$
V \sim \frac{1}{\mu_{\text {out }}} \frac{H^{3}}{(H R)} P .
$$

On eliminating $P$ between Eqs. (5) and (6), we have the desired result for the variations of the scaling for the air gap $H$, between the drop and the substrate cast in terms of the capillary number $\mathrm{Ca}=\mu_{\text {out }} V / \sigma$, the Eötvös (or Bond) number Eo $=\rho_{\text {in }} g R^{2} / \sigma$ [22], the Stokes number $\mathrm{St}=$ $\rho_{\text {in }} V R / \mu_{\text {out }}$, and the Weber number $\mathrm{We}=\rho_{\text {in }} R V^{2} / \sigma$, reflecting the competition between capillary, gravitational, and inertial forces subsumed in a composite dimensionless number, $K$,

$$
\frac{H}{R} \sim \frac{1}{\sqrt{1 / \mathrm{Ca}+\mathrm{St}+\mathrm{Eo} / \mathrm{Ca}}}=\sqrt{\frac{\mathrm{Ca}}{1+\mathrm{We}+\mathrm{Eo}}} \equiv \sqrt{K} .
$$

For millimetric drops of water or organic liquids $\mathrm{Eo} / \mathrm{Ca} \sim 0.1$, so even though drop impact is driven by gravity, its effect on deforming the drop during motion is small. For low-impact velocities where $\mathrm{We} \ll 1$, the characteristic film thickness scales as $H / R \sim \mathrm{Ca}^{1 / 2}$, whereas for high-impact velocities with $\mathrm{We} \gg 1$, $H / R \sim \mathrm{St}^{-1 / 2}$. The transition between the low- and highimpact velocity regimes is embodied in Eq. (7).

Following earlier work on the modeling of quasistatic drop impact, we can scale equations (1), (2), and (4) into nondimensional form, denoted by quantities with an overbar, using the scalings $p=P \bar{p}, \quad h=(\sqrt{2 K} R) \bar{h}$, $r=\left(2^{3 / 4} K^{1 / 4} R\right) \bar{r}$, and $t=(\sqrt{2 K} R / V) \bar{t}$. The scaled Stokes-Reynolds equation (4) becomes

$$
\frac{\partial \bar{h}}{\partial \bar{t}}=\frac{1}{12 \bar{r}} \frac{\partial}{\partial \bar{r}}\left(\bar{r} \bar{h}^{3} \frac{\partial \bar{p}_{f}}{\partial \bar{r}}\right)
$$

and the scaled equation (2) is

$$
\frac{1}{2 \bar{r}} \frac{\partial}{\partial \bar{r}}\left(\bar{r} \frac{\partial \bar{h}}{\partial \bar{r}}\right)=\bar{p}_{B}-\bar{p}_{f} .
$$

Klaseboer et al. [23] observed that for slow, quasistatic collisions (We $\ll 1$, Eo $\ll 1$ and so $\bar{p}_{B} \simeq 2$ ), the resulting universal form of Eqs. (8) and (9), with boundary condition $d h / d t=-V$ (i.e., $d \bar{h} / d \bar{t}=-1$ ), no longer depends on any physical parameter and reported that the film thickness at which a dimple first develops is given by $\bar{h}_{d} \approx 0.4$ or $h_{d} / R \approx 0.4(2 \mathrm{Ca})^{1 / 2}$. In Fig. 2, we see that this relation holds for quasistatic collisions in a variety of experimental systems in the literature: ethanol drops in air against a glass surface [14], mercury drop in water against a mica surface [24], bubble in water against a glass surface for immobile $[25,26]$ and mobile [27] film drainage, in which for mobile 


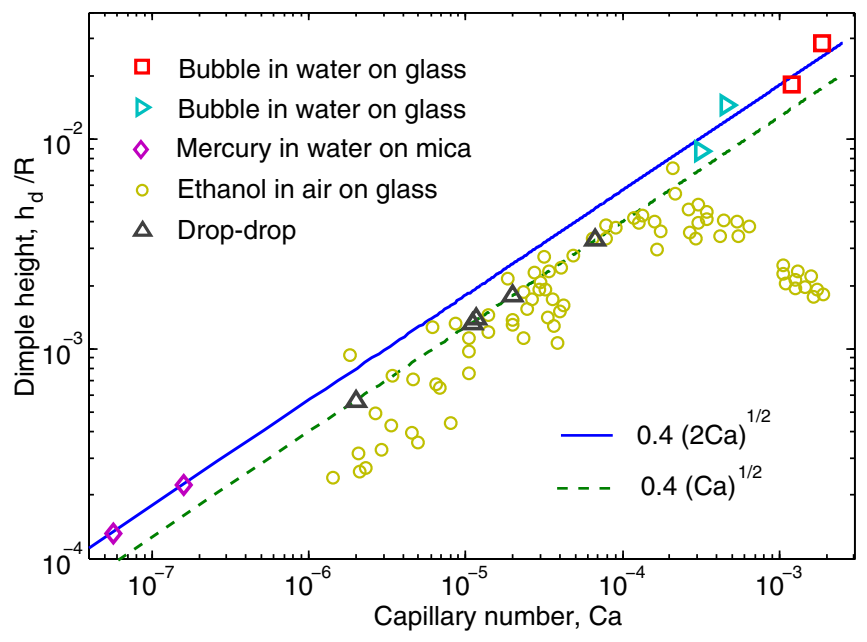

FIG. 2 (color online). Comparison of the variation of dimple height with capillary number for quasisteady drop impact. Details of the experimental systems are given in the Supplemental Material [17].

films, the numerical factor 12 in Eq. (4) is replaced by 3. For the different combinations of two liquid drops in another immiscible liquid, the relationship differs by a numerical factor: $h_{d} / R \approx 0.4 \mathrm{Ca}^{1 / 2}[23,28]$. Details of all the different experimental systems are summarized in the Supplemental Material [17]. With the exception of ethanol drops in air against glass, there is good universal agreement with the predicted $\mathrm{Ca}^{1 / 2}$ behavior.

Two factors account for deviations of the data for ethanol drops in air impacting on a glass surface from the $\mathrm{Ca}^{1 / 2}$ scaling law in Fig. 2. The experimental results is actually the central thickness just prior to film rupture and is, therefore, smaller than the film thickness at which the dimple first develops. Also at higher $\mathrm{Ca}$, the drop velocities are up to $3 \mathrm{~m} / \mathrm{s}$, corresponding to Stokes number St $\sim 10^{5}$, well beyond the quasistatic collision regime [14]. Thus, the scaling in Eq. (7) suggest the more general relationship would hold at both low- and high-impact velocities

$$
\frac{h_{d}}{R}=0.4(2 K)^{1 / 2}=0.4 \sqrt{\frac{2 \mathrm{Ca}}{1+\mathrm{We}+\mathrm{Eo}}} .
$$

Within measurement variations, the prediction based on Eq. (10) is in agreement with the ethanol drop data [14] at all impact velocities (Fig. 3).

The physical origin of the maximum in $h_{d} / R$ as a function of velocity can be understood from the normal stress balance (2). At small impact velocities, inertial effects are small and the pressure $p_{B}$ at the bottom of the drop can be approximated by $2 \sigma / R$, the Laplace pressure of the undeformed drop. The development of a dimple corresponds to an inversion of the curvature of the film, and that occurs when the pressure in the film $p_{f}$ exceeds $p_{B}$. Thus, $h_{d}$ exhibits a universal dependence on

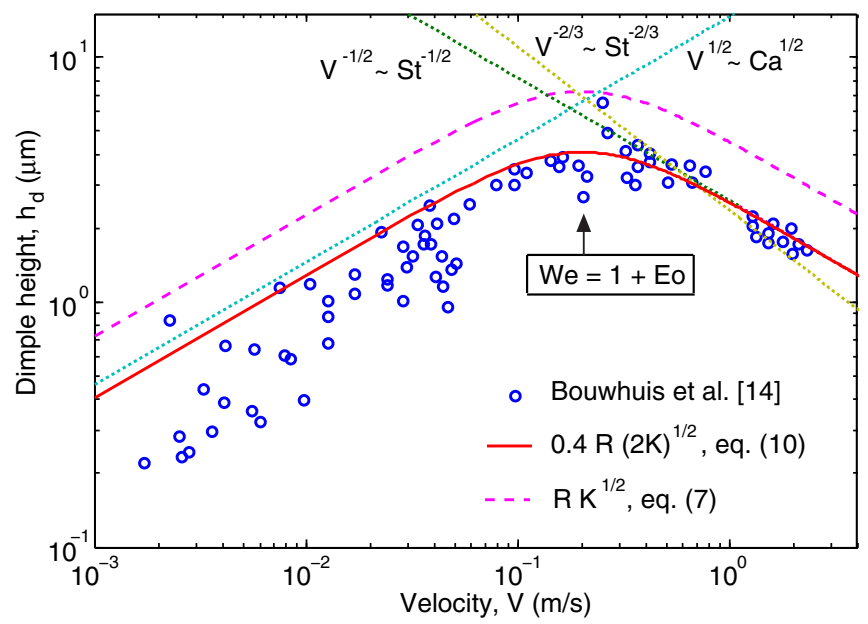

FIG. 3 (color online). Variation of dimple height with drop impact velocity for ethanol in air onto glass [14] compared to Eq. (10) (solid line) and the relation $h_{d}=R \sqrt{K}$ (dashed line) with $R=0.9 \mathrm{~mm}$. The maximum occurs at $\mathrm{We}=1+$ Eo.

the capillary number $\mathrm{Ca}$ as seen in Fig. 2. As the impact velocity increases, $p_{B}$ increases due to the stagnation pressure contribution $\frac{1}{2} \rho_{\text {in }} V^{2}$, and this has a similar effect to increasing the Laplace pressure of the drop or the effective interfacial tension, making the drop more difficult to deform. This, therefore, accounts for the decrease in $h_{d}$ at higher impact velocities seen in Fig. 3. As we shall see, this physical interpretation provides the basis for obtaining an accurate approximate solution.

Beyond the maximum in Fig. $3, h_{d}$ scales with the velocity as $V^{-1 / 2}$ or as $\mathrm{St}^{-1 / 2}$. Earlier theoretical arguments have suggested instead a $\mathrm{St}^{-2 / 3}$ scaling $[14,16]$. The difference is due to the choice of the pressure scale inside the drop at impact. Our pressure scale of Eq. (1) is the wellknown stagnation pressure since the bottom of the drop has effectively decelerated to zero velocity at impact whereas the scaling in Ref. [14] is based on an assumed scaling of the velocity potential at the bottom of the drop. The numerical evidence adduced to support the $\mathrm{St}^{-2 / 3}$ scaling was from solutions of governing equations that are different from the equations used here [14]. In any case, the available experimental data do not extend to a high enough impact velocity to differentiate between a $\mathrm{St}^{-1 / 2}$ or $\mathrm{St}^{-2 / 3}$ dependence. This open question requires further experiments for resolution.

Point mass model. - In the quasistatic low-velocity limit, evolution of the film profile $h(r, t)$ can be found by solving Eqs. (8) and (9) with $\bar{p}_{B}=2$, after eliminating the film pressure $\bar{p}_{f}$. At finite drop impact velocities, $\bar{p}_{B}$ in Eq. (9) has to be found by solving the Laplace equation for the velocity potential (Bernoulli equation) that describes potential flow in a moving domain that is the drop boundary (Supplemental Material [17]). Here, we propose a simplification by treating the drop as point particle of mass 


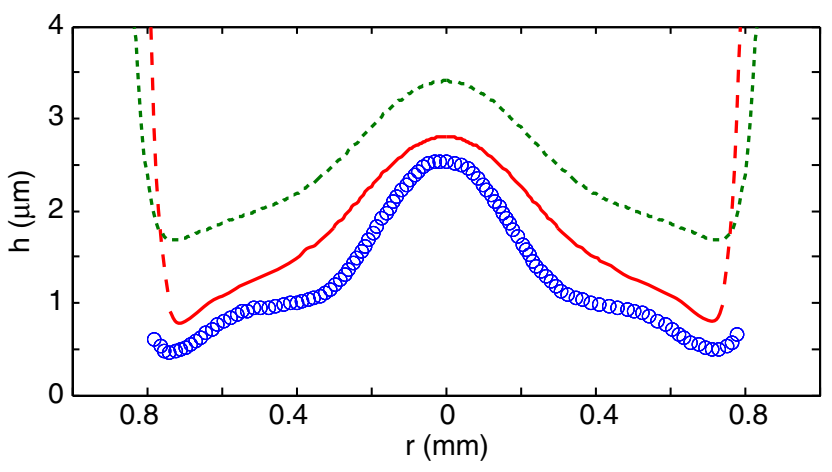

FIG. 4 (color online). Experimental film profile for a water drop attaining an impact speed of $0.22 \mathrm{~cm} / \mathrm{s}$ (circles) at the moment of maximum spreading [10] compared to predictions of the point mass model with (solid line) and without (dashed line) the inertial term, $\frac{1}{2} \rho_{\text {in }} U^{2}$, in the pressure $p_{B}$.

$m=4 \pi \rho_{\text {in }} R^{3} / 3$. The velocity of the drop is then described by the following equation of motion as it approaches the surface,

$$
m \frac{d U}{d t}=m g-\frac{\pi}{2} R^{2} C_{d} \rho_{\text {out }} U^{2}-2 \pi \int_{0}^{\infty} r p_{f} d r .
$$

The initial condition is $U=U_{o}$, and the air drag on the drop is described by the Schiller-Naumann [29] drag coefficient $C_{d}=(24 / \mathrm{Re})\left(1+0.15 \mathrm{Re}^{0.687}\right)$ that depends on the Reynolds number, $\operatorname{Re}=2 R \rho_{\text {out }} U / \mu_{\text {out }}$. The drop velocity provides a boundary condition to Eq. (8) in the form $\partial h / \partial t=-U$ at $r=r_{\max }$ where $\bar{h} \sim \bar{r}^{2}$. The point mass equation of motion (11) is coupled to the normal stress balance (2) and the Stokes-Reynolds (4) equations via the pressure $p_{B}$ that we approximate by $p_{B} \approx$ $2 \sigma / R+\frac{1}{2} \rho_{\text {in }} U^{2}$, after setting $p_{o}=0$ and omitting the small gravitational contribution, $\rho_{\text {in }} g z_{B}$ in Eq. (1).

In Fig. 4, we compare the film profile predicted by the point mass model of Eq. (11) with and without the inertial term. Since the time of the experimental film profile was not reported in Ref. [10], we can compare the film shape predicted by the model at the maximum radial extent. The agreement is quite reasonable. Note that the film radius of $a \sim 0.8 \mathrm{~mm}$ is comparable to the undeformed drop radius of $R \sim 1.0 \mathrm{~mm}$, indicative of significant deformation of the drop. As a rough estimate, if we assume all the kinetic energy of the incoming drop has all been expended to increase the surface area of the drop that has deformed under constant volume into a truncated sphere of radius $R_{t}>R$, and with the base radius $a$, the value of $a$ would be comparable to the initial drop radius $R$.

Conclusions. - We have shown that the initial deformation characteristics for drop impact can be captured by a simple analytic formula, Eq. (10), derived from scaling arguments as a generalization of an earlier result [23] for slow collisions. Quantitative comparison with a variety of experiments suggests that this scaling has captured successfully the competition between capillary, gravity, and inertia forces. For a millimetric drop, gravitational effects are not significant. Therefore, at low drop velocities, capillary forces dominate and the deformation is determined by the capillary number $\mathrm{Ca}^{1 / 2}=\left(\mu_{\text {out }} V / \sigma\right)^{1 / 2}$, but at high velocities, inertia forces dominate and the deformation is determined by the Stokes number $\mathrm{St}^{-1 / 2}=$ $\left(\rho_{\text {in }} V R / \mu_{\text {out }}\right)^{-1 / 2}$. This differs from the $\mathrm{St}^{-2 / 3}$ behavior suggested earlier due to a different estimate of the pressure at the bottom of the drop at impact. The transition between these two regimes occurs when the Weber and Eövös numbers satisfy $\mathrm{We}=1+$ Eo. With the use of a point mass model for the drop, the profile of the air film between the base of the drop and the substrate can be predicted, thus verifying that inertia at high impact velocities has the effect of increasing the effective Laplace pressure of the drop or increasing the effective surface tension, thereby reducing the extent of drop deformation.

This work was supported in part by the Particulate Fluids Processing Center, University of Melbourne and the Australian Research Council through a Discovery Project Grant to D. Y. C. C.

*evert@ihpc.a-star.edu.sg

†manicar@ihpc.a-star.edu.sg

${ }^{*}$ Visiting scientist to the IHPC when this work was initiated. D.Chan@unimelb.edu.au

[1] A. M. Worthington, Proc. R. Soc. London 25, 261 (1876).

[2] S. T. Thoroddsen, T. G. Etoh, K. Takehara, N. Ootsuka, and Y. Hatsuki, J. Fluid Mech. 545, 203 (2005).

[3] A. Gopinath and D. L. Koch, J. Fluid Mech. 454, 145 (2002).

[4] R. D. Schroll, C. Josserand, S. Zaleski, and W. W. Zhang, Phys. Rev. Lett. 104, 034504 (2010).

[5] J. Eggers, M. A. Fontelos, C. Josserand, and S. Zaleski, Phys. Fluids 22, 062101 (2010).

[6] X. G. Zhang and O. A. Basaran, J. Colloid Interface Sci. 187, 166 (1997).

[7] V. Bergeron, D. Bonn, J. Y. Martin, and L. Vovelle, Nature (London) 405, 772 (2000).

[8] L. Xu, W. W. Zhang, and S. R. Nagel, Phys. Rev. Lett. 94, 184505 (2005).

[9] M. M. Driscoll and S. R. Nagel, Phys. Rev. Lett. 107, 154502 (2011).

[10] R. C. A. van der Veen, T. Tran, D. Lohse, and C. Sun, Phys. Rev. E 85, 026315 (2012).

[11] J. de Ruiter, J. M. Oh, D. van den Ende, and F. Mugele, Phys. Rev. Lett. 108, 074505 (2012).

[12] T. Tran, H. J. J. Staat, A. Prosperetti, C. Sun, and D. Lohse, Phys. Rev. Lett. 108, 036101 (2012).

[13] J. M. Kolinski, S. M. Rubinstein, S. Mandre, M. P. Brenner, D. A. Weitz, and L. Mahadevan, Phys. Rev. Lett. 108, 074503 (2012).

[14] W. Bouwhuis, R. C. A. van der Veen, T. Tran, D. L. Keij, K. G. Winkels, I. R. Peters, D. van der Meer, C. Sun, 
J. H. Snoeijer, and D. Lohse, Phys. Rev. Lett. 109, 264501 (2012).

[15] V. Mehdi-Nejad, J. Mostaghimi, and S. Chandra, Phys. Fluids 15, 173 (2003).

[16] S. Mandre, M. Mani, and M. P. Brenner, Phys. Rev. Lett. 102, 134502 (2009); M. Mani, S. Mandre, and M. P. Brenner, J. Fluid Mech. 647, 163 (2010).

[17] See the Supplemental Material at http://link.aps.org/ supplemental/10.1103/PhysRevLett.113.194501 for [brief description].

[18] L. J. Clancy, Aerodynamics (Pitman, London, 1975).

[19] D. Y. C. Chan, E. Klaseboer, and R. Manica, Soft Matter 7, 2235 (2011).

[20] S. G. Yiantsios and R. H. Davis, J. Fluid Mech. 217, 547 (1990).

[21] Y. Amarouchene, G. Cristobal, and H. Kellay, Phys. Rev. Lett. 87, 206104 (2001).
[22] In general, one should use $\Delta \rho=\left|\rho_{\text {in }}-\rho_{\text {out }}\right|$ in the definition of the Eötvös or Bond numbers.

[23] E. Klaseboer, J. P. Chevaillier, C. Gourdon, and O. Masbernat, J. Colloid Interface Sci. 229, 274 (2000).

[24] J. N. Connor and R. G. Horn, Faraday Discuss. 123, 193 (2003).

[25] M. H. W. Hendrix, R. Manica, E. Klaseboer, D. Y. C. Chan, and C.-D. Ohl, Phys. Rev. Lett. 108, 247803 (2012).

[26] R. Manica, M. H. W. Hendrix, R. Gupta, E. Klaseboer, C.-D. Ohl, and D. Y. C. Chan, Appl. Math. Model. 38, 4249 (2014).

[27] R. Manica, M. H. W. Hendrix, R. Gupta, E. Klaseboer, C.-D. Ohl, and D. Y. C. Chan, Soft Matter 9, 9755 (2013).

[28] R. Manica, E. Klaseboer, and D. Y. C. Chan, Soft Matter 4, 1613 (2008).

[29] L. Schiller and A. Naumann, Zeit. Ver. Deut. Ing. 77, 318 (1933); See R. Clift, J. R. Grace, and M. E. Weber, Bubbles, Drops and Particles (Academic Press, New York, 1978). 